\title{
Review
}

\section{Key toolkits of non-pharmacological management in COPD: during and beyond COVID-19}

\author{
Jaber S. Alqahtani ${ }^{1,2, *}$, Saeed M Alghamdi ${ }^{3,4}$, Abdulelah M Aldhahir ${ }^{5}$, Malik Althobiani ${ }^{2,6}$, \\ Tope Oyelade ${ }^{7}$ \\ ${ }^{1}$ Department of Respiratory Care, Prince Sultan Military College of Health Sciences, 34313 Dammam, Saudi Arabia, \\ ${ }^{2}$ UCL Respiratory, University College London, WC1E 6BT London, UK, ${ }^{3}$ National Heart and Lung Initiation, Imperial \\ College London, SW7 2BX London, UK, ${ }^{4}$ Faculty of Applied Medical Sciences, Umm Al-Qura University, 21961 Makkah, \\ Saudi Arabia, ${ }^{5}$ Respiratory Care Department, Faculty of Applied Medical Sciences, Jazan University, 45142 Jazan, Saudi \\ Arabia, ${ }^{6}$ Department of Respiratory Therapy, King Abdulaziz University, 21589 Jeddah, Saudi Arabia, ${ }^{7}$ Institute for Liver \\ and Digestive Health, Division of Medicine, University College London, NW3 2PF London, UK
}

\section{TABLE OF CONTENTS}

\author{
1. Abstract \\ 2. Introduction \\ 3. Smoking cessation \\ 4. Pulmonary rehabilitation (PR) \\ 5. Telehealth applications \\ 6. Clinical implications and recommendations \\ 7. Conclusions \\ 8. Author contributions \\ 9. Ethics approval and consent to participate \\ 10. Acknowledgment \\ 11. Funding \\ 12. Conflict of interest \\ 13. References
}

\section{Abstract}

Individuals with COPD are at higher risk of severe disease and mortality if they contract COVID-19. Shielding and social distancing have negatively impacted the delivery of routine care for COPD patients, which should be maintained to avoid further deterioration. We aimed to review the literature about the key toolkits of non-pharmacological treatments of COPD patients before and during the COVID19 pandemic. In particular, we focused on smoking cessation, pulmonary rehabilitation, and telehealth delivery approaches during the COVID-19 crisis. Smoking cessation services are important to mitigate the spread of the virus, especially in people with chronic lung disease; the pandemic, in one way or another, has helped to enhance people's motivation to quit smoking. Also, tele-rehabilitation is considered as effective as conventional pulmonary rehabilitation in controlling symptoms of disease, promoting physical activity, and enhancing self-management of COPD. Telerehabilitation offers flexibility and it could be the dominant mode for providing a pulmonary rehabilitation programme.
Finally, the use of telehealth (TH) modes has trended during the pandemic. Consensus about the effectiveness of $\mathrm{TH}$ in reducing exacerbation events is still inconclusive. In the context of COPD, further clinical research must concentrate on understanding attitudes, behaviours, and motivations towards smoking cessation. Further recommendations include gauging the feasibility of a long-term telerehabilitation programme in large COPD populations, designing more COPD-related mobile apps, and evaluating the feasibility of tele-rehabilitation in clinical practice.

\section{Introduction}

Since the emergence of novel coronavirus disease 2019 (COVID-19) in Wuhan City, Hubei Province of China, which was declared a global health emergency by the World Health Organization (WHO), around 2 million people have died and many millions more have been infected [1]. COVID-19 is caused by severe acute respiratory syndrome coronavirus 2 (SARS-CoV-2), which is a novel 
strain of coronavirus that is genetically related to the severe acute respiratory syndrome (SARS) and Middle East respiratory syndrome (MERS) [2, 3]. The virus manifests with wide clinical presentations including but not limited to fever, fatigue, diarrhoea, and dry cough as the most common [4]. Several trials have investigated the effect of multiple medications and newly developed vaccines, yielding conflicting results $[5,6]$. As the number of COVID-19 cases continue to rise, the global healthcare systems are being pushed to their limits with various limitations hindering the clinical management of patients [7-9].

The Global Initiative defines chronic obstructive pulmonary disease (COPD) for Chronic Obstructive Lung Disease (GOLD) as “a common, preventable, and treatable disease that is characterised by persistent respiratory symptoms and irreversible airflow limitation that is due to airway and/or alveolar abnormalities usually caused by significant exposure to noxious particles or gases" [10]. COPD is considered to be the fourth leading cause of mortality worldwide and was responsible for the deaths of more than 3 million people worldwide in 2015 (WHO report). Without further interventions, COPD is expected to be the third leading cause of death by 2030 [11-13]. The prevalence of COPD was estimated to be $7.6 \%$ globally, with 251 million cases reported globally in 2016 [14]. Patients with COPD tend to have daily symptoms of dyspnoea, cough with or without sputum production, wheezing, and chest tightness. They also are susceptible to worsening of their symptoms or frequent exacerbations that can lead to frequent admission and readmission to hospital [15-18]. COPD exacerbation is defined as an 'acute worsening of respiratory symptoms that results in additional therapy' [10]. According to Alqahtani et al. [19] in a systematic review conducted in 2020 that included 15 studies, COVID-19 was reported to have a higher risk of severity and mortality in COPD patients. COPD exacerbations are more frequently induced by viral infections, such as coronaviruses [20, 21]. Therefore, shielding strategies have been introduced and promoted for individuals with COPD, which involve hand washing and strict social distancing to minimise the possibility of highrisk groups getting infected with COVID-19. A 50\% reduction in severe COPD exacerbations has been reported in association with COVID-19 pandemic restrictions, which supports the inclusion of respiratory virus infection control measures in future iterations of COPD guidelines [22].

The COPD 'value pyramid', which has been developed by the London Respiratory Team as a costeffective treatment approach, includes smoking cessation, pulmonary rehabilitation (PR) and telehealth [23]. However, due to the COVID-19 pandemic, the delivery of nonpharmacological management approaches has been profoundly altered around the world. Therefore, this paper aims to summarise and evaluate key toolkits of common non-pharmacological treatments with COPD during the COVID-19 pandemic. This review focuses on smoking cessation services, pulmonary rehabilitation programmes, and telehealth modes during the COVID-19 pandemic.

\section{Smoking cessation}

Evidence suggests that smokers with COPD are more susceptible to contracting COVID-19 than nonsmokers [19, 24]. Smokers with COPD are also more likely to develop serious forms of COVID-19 and have a higher mortality rate [19]. This might be due to underlying biological causes such as damage to the airway epithelial cells and upregulation of ACE2 receptors, or it could be due to behavioural causes such as hand-to-face movements, which would speed up the viral transmission [25, 26]. Related to that is the unfortunate discovery that COVID-19 pandemic-related stress has increased the number of current smokers and makes quitting exceedingly difficult for current smokers [26-28]. The limited information available on smoking cessation strategies among COPD patients during COVID-19 suggests that somking cessation might minimise the risk of acquiring the virus, developing lifethreatening complications, and/or admission to intensive care units [19, 26, 29].

Motivations and perceptions regarding smoking cessation during the COVID-19 pandemic are varied. A recent survey among 957 smokers reported that the serious concerns about acquiring COVID-19 and becoming severely vulnerable were the motivations to stop smoking or attempting to stop smoking [27]. The study also evaluated the relationship between smoking and stress-related COVID-19, the perceived difficulty of quitting smoking due to the pandemic, and the motivations to quit smoking [27]. The study reported that current smokers had a significant reduction in smoking habits during the pandemic compared to that before the pandemic $(\mathrm{OR}=1.80$; $95 \% \mathrm{CI}$ : 1.07-3.05; $p=0.027$ ) [27].

Further, the authors highlighted the most common motivations for quitting smoking among participants, which were living a healthier life (32\%), being alone, avoiding social gatherings (27\%), having healthier lungs (21\%), and having better recovery outcomes from COVID19 (19\%). None of the motivations indicated that smokers needed to quit smoking because they wished to do so. In some ways, the pandemic has created an opportunity for many smokers with or without long-term lung conditions to change their behaviours towards smoking and/or to attempt to increase their motivation to quit all types of smoking, as demonstrated by British Lung Foundation Survey data [30]. The survey included 7975 participants with a long-term lung condition. This data showed that $65 \%$ of the participants attempted to quit tobacco smoking during the pandemic [30]. Related to that, the Action on Smoking and Health (Ash) organisation reported that a million smokers in the UK quit smoking during COVID-19, and an additional 550,000 have attempted to stop smoking, while 2.4 
million reduced the number of cigarettes they smoke [31]. A recent study in the US examined participants' intentions to quit smoking in the next 6 months due to COVID-19. The study included 777 smoker and non-smoker participants. The smokers perceived that they are at a higher risk of complications from COVID-19 compared to non-smokers (76\%). The majority of the participants (71\%) intended to stop smoking in the next six months of the pandemic, and half of the participants (50\%) reported attempting to quit since the pandemic started [32]. Increasing smoking quit rate has the potential to reduce SARS-CoV-2 transmission and the associated poor prognosis; therefore it should be widely endorsed [33]. All in all, high quality smoking cessation trials that include unconventional strategies should be integral part of public health efforts in order to lessen the smoking burden on society.

\section{Pulmonary rehabilitation (PR)}

PR has been defined by the European Respiratory Society (ERS) and American Thoracic Society (ATS) as 'an evidence-based, multidisciplinary and comprehensive intervention for patients with chronic respiratory diseases who are symptomatic and often have decreased daily life activities' [34]. PR has been shown to be effective in reducing exacerbation, hospitalisation, emergency department visits, symptoms of dyspnoea, anxiety and depression, leg discomfort, and healthcare costs [34-38]. PR also improves exercise capacity, emotional function, muscle strength and endurance, health-related quality of life, nutritional status, and disease self-management in stable COPD patients [3941]. The benefits of PR can be obtained despite the patient's gender, age, smoking status, disease severity, as well as it can be maintained for 12 months in those who have attended PR before [34-38, 42]. PR consists of patient assessment, exercise training, education, nutritional counselling, and psychological counselling. It is administered by a multidisciplinary team that includes doctors, physiotherapists, nurses, psychologists, dietitians or nutritionists, occupational therapists, social workers and psychologists [43-46]. PR modes are vary but the duration is recommended to last from 6 to 12 weeks of supervised sessions, and patients are advised to attend at least twice per week [47].

There is rising evidence that $\mathrm{PR}$ is safe and effective during and after hospitalisation due to AECOPD [48]. Given the evidence that PR is effective with stable COPD patients, several studies have investigated the effect of PR during and after hospitalisation due to AECOPD. Seymour et al. [49] examined the influence of outpatient PR following exacerbation in reducing re-exacerbation that required hospital admission among 60 COPD patients. They found that PR was associated with a reduced frequency of reexacerbation requiring hospitalisation or emergency visits in the following three months. Man et al. [50] evaluated the effect of PR on exercise capacity measured by incremental shuttle walk distance (ISWD) and health status following hospitalisation due to AECOPD in 42 COPD patients for three months. They concluded that PR significantly improved exercise capacity and health status at the three-month point in the early PR group when compared with the usual care group [50]. A recent systematic review and meta-analysis conducted by Puhan et al. [51], which included 20 studies and 1477 participants, concluded that early PR following COPD exacerbation improved healthrelated quality of life and exercise capacity in COPD patients.

Predominantly, PR is administered as a centrebased programme, inpatient or outpatient, where individuals are assessed and monitored by a multi-professional team. However, this mode of delivery has been heavily affected by the COVID-19 pandemic [52]. Centre-based PR programmes in which groups of COPD patients gather together have been suspended globally due to staff redeployment to acute settings, and to ensure that effective shielding and social distancing occur, whereas the need for PR still exists. Home-based PR approaches facilitated through digital technologies such as tele-PR have been proposed and tested against conventional PR in the COPD population. Hansen et al. [53] investigated whether a ten-week course of Tele-PR is superior to conventional PR by measuring exercise capacity, respiratory symptoms, health-related quality of life, physical activity and lower limb muscle function in patients with 134 stable COPD patients. They concluded that more participants completed the tele-PR programme and there were no differences between groups in all outcome measures.

The feasibility of tele-PR has been examined by Chaplin et al. [54] in a randomised clinical trial that included 103 stable COPD patients who attended conventional rehabilitation or tele-rehabilitation. The researchers found that the tele-PR programme is feasible and acceptable compared to a conventional rehabilitation programme. Thus, using tele-PR during the COVID-19 pandemic may be an essential way to maintain the existence of PR, since COPD patients are at a higher risk of mortality [19]. Several PR-service applications have been introduced and their efficiency has been tested through, for example, 'myPR', 'SPACE' and video conferencing [54, 55]. During tele-PR, patients are provided with self-explanatory exercises and educational materials for the entire programme that can be accessed at the patients' convenience, without the need for the virtual presence of healthcare providers. Other forms of tele-PR are run by home teleconferencing through 'Zoom' where a healthcare provider is virtually present and provides live exercise and educational sessions that take place at the patient's home [56]. To assess exercise capacity remotely after the end of tele-rehabilitation, Houchen-Wolloff et al. conducted a systematic review that included 16 studies and concluded that four-metre gait speed (4MGS) had 
the best correlation with routine measures of exercise capacity. The incremental shuttle walk test (ISWT) and sixminute walk test (6MWT) were implemented during faceto-face PR programmes [57]. The long-term effects of the COVID-19 pandemic on the availability of face to face services is unknown, therefore, there is a need for accelerated implementation of new ways of sustaining the benefits of COVID-related-PR . This would be a good approach if we could do this for our patients remotely.

\section{Telehealth applications}

WHO has defined TH as "delivery of health care services, where patients and providers are separated by distance”. Without doubt, TH tools have become more mainstream than in the past due to the pandemic [58]. In the context of COPD, there is widespread international use of $\mathrm{TH}$ in COPD which, can offer an opportunity to protect extremely vulnerable patients from acquiring the virus [59]. At the organisational level, it also offers different modes to monitor and deliver routine care, virtual pulmonary rehabilitation, and emotional support during the pandemic [56, 60]. Taking into consideration the lack of relevant data about the effectiveness of $\mathrm{TH}$ in treating COPD during the pandemic, there is a consensus in the current guidelines about minimising face-to-face contact and encouraging TH applications, especially among vulnerable patients such as those with COPD [61, 62]. Patients with COPD are considered high-risk, and it has been recognised in the literature that the TH applications that have been introduced help these people to optimise their health, limit the risk of acquiring the infection, decelerate exacerbation events, and receive the necessary care to minimise hospital admissions $[30,63]$. Besides, TH seems to reduce the number of exacerbations, as reported in the clinical studies [63-65]. This reduction in exacerbations was not particularly due to improvements in lung conditions, but it was mostly as a result of the shielding strategies and the wide range of public restrictions (e.g., hand hygiene, universal masking) [63, 64]. In clinical practice, $\mathrm{TH}$ during the pandemic was found to be effective in reducing hospital admissions due to exacerbations and it was noted that most of the participants117/153 (76\%) - had a low burden of COPD symptoms [63]. A UK survey reported that $90 \%$ of the participants with chronic lung conditions were actively shielding and using tele-services to receive care [30]. However, TH services are yet to fulfil expectations, which has led to a high demand for the development of simpler and more interactive TH applications that consider the behaviours and wellbeing of patients with COPD. A recent study in the UK evaluated the change in treatment for COPD patients before and during the lockdown. The study detected an increase in exacerbation events, anxiety, depression, and behavioural changes [65]. Better prevention of COPD exacerbations has been listed as the top priority for COPD exacerbation research in a recent patient-clinician research prioritisation [66]. In clinical practice, many mobile phone apps have been launched to track symptoms of COVID-19 or potential exposure, to receive recommendations, and to notify the public about infected locations [56, 67]. In general, these apps have high acceptance and download rates due to encouragement from governments to use mobile apps to mitigate the pandemic [56, 67]. The long-term TH acceptance rate must be investigated to understand whether people believe that TH would continue as a mainstream mode for care delivery even beyond the pandemic, particularly in monitoring lung conditions such as COPD.

In chronic health conditions, the attitude towards using mobile apps was explored in a study that involved 10790 participants. This study aimed to evaluate the associations between chronic lung conditions (including COPD) and attitudes towards TH apps. In general, the study found that around $22 \%$ of the participants were likely to use the $\mathrm{TH}$ app to track symptoms and receive recommendations. Moreover, 24\% of the participants were likely to use $\mathrm{TH}$ apps to avoid infected locations. Overall, people with severe chronic lung diseases used TH apps more often than those without chronic lung diseases (OR and 95\% CI: 1.23, 1.04 to 1.45 ) [68]. A recent study reported that clinicians were able to evaluate symptom severity (95\%), promote smoking cessation (84\%) and signpost to other healthcare resources (80\%) [69]. Also, patients involved in the study reported that assessment of COPD severity and starting new prescriptions were being fully addressed via $\mathrm{TH}$. This highlights the importance of using TH to deliver patients' health care, but still using such services have some limitations that need to be considered and improved over time even after the COVID-19 pandemic recedes.

\section{Clinical implications and recommendations}

In the context of smoking cessation during the pandemic, it is important to ensure that there is easy access to smoking cessation services to maximise the chance of success in quitting smoking [30]. Also, we need more epidemiological data to understand the relationship between nicotine exposure and COVID-19 complications and COPD exacerbations. Furthermore, the pandemic could be the prime time to implement smoking cessation strategies since more current smokers are open to changing their smoking behaviours. Added to that, smoking cessation strategies could be offered by TH tools, which are considered as the most convenient methods during the pandemic, and/or the opportunity could be taken to produce more interactive smoking cessation apps to promote smoking cessation [70].

Pulmonary rehabilitation for COPD patients should be resumed despite the existence of the COVID-19 pandemic. This approach might be considered a choice to enhance PR access and adherence even beyond this crisis. To do so, face-to-face rehabilitation may be replaced with 
tele-rehabilitation, which has proven to be an effective and safe alternative approach with COPD individuals. However, further research is needed to assess the feasibility, reliability and efficacy of providing virtual/home rehabilitation.

It has been clear that using $\mathrm{TH}$ in the pandemic is vital for people with chronic lung conditions. However, there is a pressing need to develop specific TH tools and mobile apps for COPD populations that consider their psychological needs, mental wellbeing, and disease management, particularly during the COVID-19 pandemic. This might lead to a significant research demand because the current COVID-19 apps were designed for the public rather than for chronically ill patients such as those with COPD. Additionally, it would be valuable if researchers could explore the COPD patient behaviours surrounding current $\mathrm{TH}$ tools and whether these help to minimise the exacerbation rate. Acceptability and feasibility of alternative modes of TH should be evaluated broadly to assess factors related to the onset and sustainability of using TH applications during COVID-19.

\section{Conclusions}

During COVID-19 pandemic, people with COPD can maintain routine care using TH applications in monitoring symptoms, tracking the onset of COVID-19, virtually attending tele-rehabilitation programmes, and receiving support to quit smoking. Smoking cessation rate has been increased during COVID-19 pandemic compared to pre-pandemic times and this has the potential to reduce SARS-CoV-2 transmission and the associated poor prognosis. Tele-PR has been proven to be an effective and safe alternative approach with COPD patients, thus using tele-PR during COVID-19 pandemic may be a necessary approach to maintain efficient intervention delivery to these patients. TH use during COVID-19 pandemic was feasible, acceptable to patients and widely used by clinicians with COPD patients, but still these services have some limitations that need to be considered and improved over time even after the COVID-19 pandemic recedes. Further work is needed to enhance TH implementations in smoking cessation and pulmonary rehabilitation.

\section{Author contributions}

JSA and SMA and AMA conceived, designed and write up the review; MA and TO analyzed the data and approved the final version; with support from JSA, SMA and AMA.

\section{Ethics approval and consent to participate}

Not applicable.

\section{Acknowledgment}

Thanks to all the peer reviewers for their opinions and suggestions.

\section{Funding}

This study received no external funding.

\section{Conflict of interest}

The authors declare no conflict of interest.

\section{References}

[1] Pelkonen M, Notkola I, Nissinen A, Tukiainen H, Koskela H. Thirty-Year Cumulative Incidence of Chronic Bronchitis and COPD in Relation to 30-Year Pulmonary Function and 40-Year Mortality. Chest. 2006; 130: 1129-1137.

[2] Su S, Wong G, Shi W, Liu J, Lai ACK, Zhou J, et al. Epidemiology, Genetic Recombination, and Pathogenesis of Coronaviruses. Trends in Microbiology. 2016; 24: 490-502.

[3] Zhu N, Zhang D, Wang W, Li X, Yang B, Song J, et al. A Novel Coronavirus from Patients with Pneumonia in China, 2019. New England Journal of Medicine. 2020; 382: 727-733.

[4] Wang Y, Wang Y, Chen Y, Qin Q. Unique epidemiological and clinical features of the emerging 2019 novel coronavirus pneumonia (COVID-19) implicate special control measures. Journal of Medical Virology. 2020; 92: 568-576.

[5] Polack FP, Thomas SJ, Kitchin N, Absalon J, Gurtman A, Lockhart S, et al. Safety and Efficacy of the BNT162b2 mRNA Covid-19 Vaccine. New England Journal of Medicine. 2020; 383: 2603-2615.

[6] Kalil AC, Patterson TF, Mehta AK, Tomashek KM, Wolfe CR, Ghazaryan V, et al. Baricitinib plus Remdesivir for Hospitalized Adults with Covid-19. New England Journal of Medicine. 2020; 384: 795-807.

[7] World Health Organization. Clinical management of COVID19: interim guidance, 27 May 2020. World Health Organization. 2020.

[8] Alqahtani JS, Mendes RG, Aldhahir A, Rowley D, AlAhmari MD, Ntoumenopoulos G, et al. Global Current Practices of Ventilatory Support Management in COVID-19 Patients: An International Survey. Journal of Multidisciplinary Healthcare. 2020; 13: 1635-1648.

[9] Mahase E. Covid-19: Pfizer and BioNTech submit vaccine for US authorisation. British Medical Journal. 2020; 371: m4552.

[10] Vogelmeier CF, Criner GJ, Martinez FJ, Anzueto A, Barnes PJ, Bourbeau J, et al. Global Strategy for the Diagnosis, Management and Prevention of Chronic Obstructive Lung Disease: 2020 Report. American Journal of Respiratory and Critical Care Medicine. 2020:1-141.

[11] Mathers CD, Loncar D. Projections of global mortality and burden of disease from 2002 to 2030. PLoS Medicine. 2006; 3: e442.

[12] Murray CJ, Lopez AD. Alternative projections of mortality and disability by cause 1990-2020: Global Burden of Disease Study. Lancet. 1997; 349: 1498-1504.

[13] May SM, Li JTC. Burden of chronic obstructive pulmonary disease: healthcare costs and beyond. Allergy and Asthma Proceedings. 2015; 36: 4-10.

[14] Raherison C, Girodet P. Epidemiology of COPD. European Respiratory Review. 2009; 18: 213-221.

[15] Janson C, Marks G, Buist S, Gnatiuc L, Gislason T, McBurnie $\mathrm{MA}$, et al. The impact of COPD on health status: findings 
from the BOLD study. European Respiratory Journal. 2013; 42: 1472-1483.

[16] Van Remoortel H, Hornikx M, Demeyer H, Langer D, Burtin C, Decramer M, et al. Daily physical activity in subjects with newly diagnosed COPD. Thorax. 2013; 68: 962-963.

[17] Alqahtani JS, Njoku CM, Bereznicki B, Wimmer BC, Peterson GM, Kinsman L, et al. Risk factors for all-cause hospital readmission following exacerbation of COPD: a systematic review and meta-analysis. European Respiratory Review. 2020; 29: 190166.

[18] Njoku CM, Alqahtani JS, Wimmer BC, Peterson GM, Kinsman L, Hurst JR, et al. Risk factors and associated outcomes of hospital readmission in COPD: a systematic review. Respiratory Medicine. 2020; 173: 105988.

[19] Alqahtani JS, Oyelade T, Aldhahir AM, Alghamdi SM, Almehmadi M, Alqahtani AS, et al. Prevalence, severity and mortality associated with COPD and smoking in patients with COVID-19: a rapid systematic review and meta-analysis. PLoS ONE. 2020; 15: e0233147.

[20] White AJ, Gompertz S, Stockley RA. Chronic obstructive pulmonary disease . 6: the aetiology of exacerbations of chronic obstructive pulmonary disease. Thorax. 2003; 58: 73-80.

[21] Wedzicha JA, Seemungal TAR. COPD exacerbations: defining their cause and prevention. Lancet. 2007; 370: 786-796.

[22] Alqahtani JS, Oyelade T, Aldhahir AM, Mendes RG, Alghamdi $\mathrm{SM}$, Miravitlles M, et al. Reduction in COPD exacerbations during COVID-19: a systematic review and meta-analysis. medRxiv. 2021.

[23] Zoumot Z, Jordan S, Hopkinson NS. Emphysema: time to say farewell to therapeutic nihilism. Thorax. 2014; 69: 973-975.

[24] Tal-Singer R, Crapo JD. COPD at the time of COVID-19: a COPD Foundation perspective. Chronic Obstructive Pulmonary Diseases. 2020; 7: 73.

[25] Seijas N. People who smoke and suffer from COPD are more vulnerable to COVID-19 complications 2020. 2020. Available at: https://www.clinicbarcelona.org/en/news/people-who-smo ke-and-suffer-from-copd-are-more-vulnerable-to-covid-19-c omplications (Accessed: 25 April 2021).

[26] Eisenberg SL, Eisenberg MJ. Smoking Cessation during the COVID-19 Epidemic. Nicotine \& Tobacco Research. 2020; 22: 1664-1665.

[27] Bommele J, Hopman P, Walters BH, Geboers C, Croes E, Fong GT, et al. The double-edged relationship between COVID-19 stress and smoking: implications for smoking cessation. Tobacco Induced Diseases. 2020;18: 63.

[28] Klemperer EM, West JC, Peasley-Miklus C, Villanti AC. Change in Tobacco and Electronic Cigarette Use and Motivation to Quit in Response to COVID-19. Nicotine \& Tobacco Research. 2020; 22: 1662-1663.

[29] Vardavas CI, Nikitara K. COVID-19 and smoking: a systematic review of the evidence. Tobacco induced diseases. 2020; 18.

[30] Philip KE, Lonergan B, Cumella A, Farrington-Douglas J, Laffan M, Hopkinson NS. COVID-19 related concerns of people with long-term respiratory conditions: a qualitative study. BMC Pulmonary Medicine. 2020; 20: 1-10.

[31] (Ash) AoSaH. E-cigarette use decreases as evidence shows they increase smokers' chances of quitting 2020. 2020. Available at: https://ash.org.uk/category/media-and-news/press-rel eases-media-and-news/ (Accessed: 15 April 2021).

[32] Kowitt SD, Cornacchione Ross J, Jarman KL, Kistler CE, Lazard AJ, Ranney LM, et al. Tobacco quit intentions and behaviors among cigar smokers in the United States in response to COVID-19. International Journal of Environmental Research and Public Health. 2020; 17: 5368.

[33] Alqahtani JS, Aldhahir AM, Oyelade T, Alghamdi SM, Almamary AS. Smoking cessation during COVID-19: the top to-do list. Npj Primary Care Respiratory Medicine. 2021; 31: 22.

[34] Gibson GJ, Loddenkemper R, Lundbäck B, Sibille Y. Respiratory health and disease in Europe: the new European lung white book. European Respiratory Journal. 2013; 42: 559-563.

[35] Janssens T, De Peuter S, Stans L, Verleden G, Troosters T, Decramer $\mathrm{M}$, et al. Dyspnea perception in COPD: association between anxiety, dyspnea-related fear, and dyspnea in a pulmonary rehabilitation program. Chest. 2011; 140: 618-625.

[36] McCarthy B, Casey D, Devane D, Murphy K, Murphy E, Lacasse Y. Pulmonary rehabilitation for chronic obstructive pulmonary disease. Cochrane Database of Systematic Reviews. 2015; CD003793.

[37] Moore E, Palmer T, Newson R, Majeed A, Quint JK, Soljak MA. Pulmonary Rehabilitation as a mechanism to reduce hospitalizations for acute exacerbations of COPD: a systematic review and meta-analysis. Chest. 2016; 150: 837-859.

[38] Paz-Díaz H, Montes de Oca M, López JM, Celli BR. Pulmonary rehabilitation improves depression, anxiety, dyspnea and health status in patients with COPD. American Journal of Physical Medicine \& Rehabilitation. 2007; 86: 30-36.

[39] Alqahtani JS, Oyelade T, Sreedharan J, Aldhahir AM, Alghamdi SM, Alrajeh AM, et al. Diagnostic and clinical values of noncardiac ultrasound in COPD: a systematic review. BMJ Open Respiratory Research. 2020; 7: e000717.

[40] Aldhahir AM, Rajeh AMA, Aldabayan YS, Drammeh S, Subbu $\mathrm{V}$, Alqahtani JS, et al. Nutritional supplementation during pulmonary rehabilitation in COPD: a systematic review. Chronic Respiratory Disease. 2020; 17: 147997312090495.

[41] Aldabayan YS, Ridsdale HA, Alrajeh AM, Aldhahir AM, Lemson A, Alqahtani JS, et al. Pulmonary rehabilitation, physical activity and aortic stiffness in COPD. Respiratory Research. 2019; 20: 166.

[42] Busby AK, Reese RL, Simon SR. Pulmonary rehabilitation maintenance interventions: a systematic review. American Journal of Health Behavior. 2014; 38: 321-330.

[43] Evans RA, Singh SJ. Minimum important difference of the incremental shuttle walk test distance in patients with COPD. Thorax. 2019; 74: 994-995.

[44] Lareau SC, Fahy B. Pulmonary Rehabilitation. American Journal of Respiratory and Critical Care Medicine. 2018; 198: P19P20.

[45] Nici L, Donner C, Wouters E, Zuwallack R, Ambrosino N, Bourbeau J, et al. American Thoracic Society/European Respiratory Society statement on pulmonary rehabilitation. American Journal of Respiratory and Critical Care Medicine. 2006; 173: 1390-1413.

[46] Wüst RCI, Degens H. Factors contributing to muscle wasting and dysfunction in COPD patients. International Journal of Chronic Obstructive Pulmonary Disease. 2007; 2: 289-300.

[47] National Heart Lung \& Blood institute. Pulmonary Rehabilitation. 2018. Available at: https://www.nhlbi.nih.gov/health-topic s/pulmonary-rehabilitation (Accessed: 10 February 2021).

[48] Machado A, Matos Silva P, Afreixo V, Caneiras C, Burtin C, Marques A. Design of pulmonary rehabilitation programmes during acute exacerbations of COPD: a systematic review and network meta-analysis. European Respiratory Review. 2020; 29: 200039.

[49] Seymour JM, Moore L, Jolley CJ, Ward K, Creasey J, Steier JS, et al. Outpatient pulmonary rehabilitation following acute exacerbations of COPD. Thorax. 2010; 65: 423-428.

[50] Man WD, Polkey MI, Donaldson N, Gray BJ, Moxham J. Community pulmonary rehabilitation after hospitalisation for acute exacerbations of chronic obstructive pulmonary disease: randomised controlled study. British Medical Journal. 2004; 329: 1209.

[51] Puhan MA, Gimeno-Santos E, Cates CJ, Troosters T. Pulmonary rehabilitation following exacerbations of chronic obstructive pulmonary disease. Cochrane Database of Systematic Reviews. 2016; 12: CD005305.

[52] Aldhahir AM, Aldabayan YS, Alqahtani JS, Ridsdale HA, Smith C, Hurst JR, et al. A double-blind randomised controlled trial of protein supplementation to enhance exercise capacity in COPD 
during pulmonary rehabilitation: a pilot study. ERJ Open Research. 2021; 7: 00077-02021.

[53] Hansen H, Bieler T, Beyer N, Kallemose T, Wilcke JT, Østergaard LM, et al. Supervised pulmonary tele-rehabilitation versus pulmonary rehabilitation in severe COPD: a randomised multicentre trial. Thorax. 2020; 75: 413-421.

[54] Chaplin E, Hewitt S, Apps L, Bankart J, Pulikottil-Jacob R, Boyce $\mathrm{S}$, et al. Interactive web-based pulmonary rehabilitation programme: a randomised controlled feasibility trial. BMJ Open. 2017; 7: e013682.

[55] Bourne S, DeVos R, North M, Chauhan A, Green B, Brown T, et al. Online versus face-to-face pulmonary rehabilitation for patients with chronic obstructive pulmonary disease: randomised controlled trial. BMJ Open. 2017; 7: e014580.

[56] Alghamdi S, Alqahtani J, Aldhahir A. Current status of telehealth in Saudi Arabia during COVID-19. Journal of Family and Community Medicine. 2020; 27: 208.

[57] Houchen-Wolloff L, Daynes E, Watt A, Chaplin E, Gardiner N, Singh S. Which functional outcome measures can we use as a surrogate for exercise capacity during remote cardiopulmonary rehabilitation assessments? A rapid narrative review. ERJ Open Research. 2020; 6: 00526-02020.

[58] Hollander JE, Carr BG. Virtually Perfect? Telemedicine for Covid-19. New England Journal of Medicine. 2020; 382: 16791681.

[59] Alrajeh AM, Aldabayan YS, Aldhair AM, Pickett E, Quaderi SA, Alqahtani JS, et al. Global use, utility, and methods of telehealth in COPD: a health care provider survey. International Journal of Chronic Obstructive Pulmonary Disease. 2019; 14: 1713-1719.

[60] Lewis A, Bevan-Smith E, Lound A, Conway J. Pulmonary telerehabilitation in the COVID-19 era. Thorax. 2020.

[61] (NICE). COVID-19 rapid guideline: managing suspected or confirmed pneumonia in adults in the community. 2020. Available at: https://www.nice.org.uk/guidance/ng165 (Accessed: 20 April 2021).

[62] GOLD. Remote copd patient follow-up during COVID-19 pandemic restrictions. 2021. Available at: https://goldcopd.org/remote-copd-patient-follow-up-during-c ovid-19-pandemic-restrictions/ (Accessed: 20 February 2021).

[63] Liang Y, Chang C, Chen Y, Dong F, Zhang L, Sun Y. Symp- toms, Management and Healthcare Utilization of COPD Patients During the COVID-19 Epidemic in Beijing. International Journal of Chronic Obstructive Pulmonary Disease. 2020; 15: 2487.

[64] Chan KPF, Ma TF, Kwok WC, Leung JKC, Chiang KY, Ho JCM, et al. Significant reduction in hospital admissions for acute exacerbation of chronic obstructive pulmonary disease in Hong Kong during coronavirus disease 2019 pandemic. Respiratory Medicine. 2020; 171: 106085.

[65] McAuley H, Hadley K, Elneima O, Brightling CE, Evans RA, Steiner MC, et al. COPD in the time of COVID-19: an analysis of acute exacerbations and reported behavioural changes in patients with COPD. ERJ Open Research. 2021; 7: 00718-02020.

[66] Alqahtani JS, Aquilina J, Bafadhel M, Bolton CE, Burgoyne T, Holmes S, et al. Research priorities for exacerbations of COPD. Lancet Respiratory Medicine. 2021; S2213-2600(21)00227-7.

[67] Chidambaram S, Erridge S, Kinross J, Purkayastha S. Observational study of UK mobile health apps for COVID-19. Lancet Digital Health. 2020; 2: e388-e390.

[68] Camacho-Rivera M, Islam JY, Rivera A, Vidot DC. Attitudes Toward Using COVID-19 mHealth Tools Among Adults With Chronic Health Conditions: Secondary Data Analysis of the COVID-19 Impact Survey. JMIR mHealth and uHealth. 2020; 8: e24693.

[69] Wu F, Burt J, Chowdhury T, Fitzpatrick R, Martin G, van der Scheer JW, et al. Specialty COPD care during COVID-19: patient and clinician perspectives on remote delivery. BMJ Open Respiratory Research. 2021; 8: e000817.

[70] Sutton S, Smith S, Jamison J, Boase S, Mason D, Prevost AT, et al. Study protocol for iQuit in Practice: a randomised controlled trial to assess the feasibility, acceptability and effectiveness of tailored web-and text-based facilitation of smoking cessation in primary care. BMC Public Health. 2013; 13: 1-9.

Keywords: Smoking cessation; COVID-19; COPD; Pulmonary rehabilitation; Telehealth; Exacerbation

Send correspondence to: Jaber S. Alqahtani, Department of Respiratory Care, Prince Sultan Military College of Health Sciences, 34313 Dammam, Saudi Arabia, UCL Respiratory, University College London, WC1E 6BT London, UK, E-mail: Alqahtani-Jaber@hotmail.com 